\title{
Measurement of the $\mathrm{J} / \psi$ photoproduction cross section close to threshold
}

\author{
Eugene Chudakov ${ }^{* \dagger}$ \\ Thomas Jefferson National Accelerator Facility \\ E-mail: gen@jlab.org \\ on behalf of the GlueX Collaboration
}

\begin{abstract}
We present preliminary results for the $\mathrm{J} / \psi$ close-to-threshold photoproduction obtained in the GlueX experiment at Jefferson Lab. Close-to-threshold photoproduction $\gamma+p \rightarrow J / \psi+p$ probes small-size gluon configurations in the proton. This reaction may also contain a contribution from the s-channel production of the LHCb pentaquark $P(4450) \rightarrow J / \psi+p$ at beam energies of about $10 \mathrm{GeV}$. Measuring the size of the contribution allows to evaluate or put a limit on the branching ratio of the decay $P \rightarrow J / \psi+p$. The GlueX experiment is in the process of collecting data. The main research topic of the experiment is the spectroscopy of light mesons produced by a linearly polarized photon beam with the end point of $\sim 12 \mathrm{GeV}$.
\end{abstract}

23rd International Spin Physics Symposium - SPIN2018 -

10-14 September, 2018

Ferrara, Italy

* Speaker.

${ }^{\dagger}$ Authored by Jefferson Science Associates, LLC under U.S. DOE Contract No. DE-AC05-06OR23177. The U.S. Government retains a non-exclusive, paid-up, irrevocable, world-wide license to publish or reproduce this manuscript for U.S. Government purposes. 


\section{Introduction}

The photoproduction $\gamma+p \rightarrow J / \psi+p$ close to threshold $\left(E_{\gamma} \approx 8.2 \mathrm{GeV}\right)$ probes small-size gluon configurations in the nucleon. The energy dependence of the cross section, known experimentally only at higher energies [1,2], has been discussed in a number of theoretical frameworks $[3,4,5,6]$. This reaction is also interesting since it may contain a contribution from the s-channel production of the LHCb pentaquark [7] $P(4450) \rightarrow J / \psi+p$ at beam energies of about $10.1 \mathrm{GeV}$. According to several theoretical papers $[8,9,10,11,12,13]$, measuring the size of this contribution would allow to evaluate, or put a limit on, the branching ratio of the decay $P \rightarrow J / \psi+p$.

The Thomas Jefferson National Accelerator Facility (aka JLab) has finished a major upgrade that doubled the energy of the electron beam to $12 \mathrm{GeV}$. The physics program [14] includes meson spectroscopy. The main research topic of the GlueX experiment running in a new experimental Hall $\mathrm{D}$ is the spectroscopy of light mesons produced by a linearly polarized photon beam with the end point of $\sim 12 \mathrm{GeV}$. The experiment ran in 2016-2018 and has nearly finished the data taking. By the time of this presentation about $20 \%$ of the full data set had been processed and analyzed. It is expected that the full data set will be analyzed by the end of 2019, and that the systematic uncertainties will also be improved by that time. The $\mathrm{J} / \psi$ photoproduction will also be studied in other experimental halls at JLab, which run at about $1 \mathrm{GeV}$ lower endpoint energy.

\section{GlueX Experiment}

\subsection{Apparatus}

The GlueX experiment is running in the Hall D complex (Fig. 1). The electron beam is extracted from the CEBAF accelerator to the Tagger Hall, where it passes through a thin $(\sim 0.04 \%$ R.L.) diamond radiator and is deflected by a dipole Tagger Magnet into the beam dump. The beam has a $250 \mathrm{MHz}$ RF structure and a spot size on the radiator of about $1 \mathrm{~mm}^{2}$. The diamond orientation provides coherent Bremsstrahlung photons, peaked at an energy of about $9 \mathrm{GeV}$. The photons in the peak are linearly polarized. In order to increase the polarization, the photon beam passes through a $5 \mathrm{~mm}$ diameter collimator, located $75 \mathrm{~m}$ downstream of the radiator. The diamond orientation is alternated during the experiment in order to optimize the asymmetry measurements ${ }^{1}$.

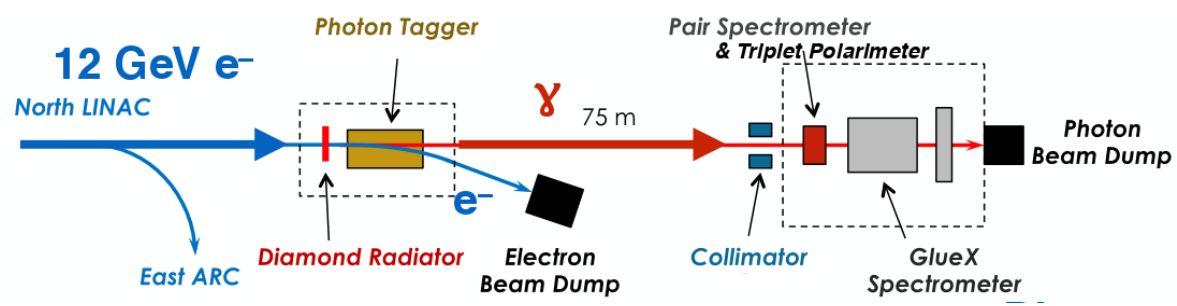

Figure 1: Hall D complex.

The electrons that radiated enough energy are detected in scintillation counters located alongside the Tagger Magnet, providing a measurement of the energy of the radiated photon with a

\footnotetext{
${ }^{1}$ The linear polarization of the beam is not used in the study reported here.
} 
resolution of about $0.1 \%$. The maximum photon energy detected is about $200 \mathrm{MeV}$ lower than the endpoint. Most of the data were collected at the electron beam energy of $11.7 \mathrm{GeV}$, but some data (in 2016) were collected at $12 \mathrm{GeV}$.

The energy spectrum and the flux of the tagged photons downstream of the collimator are measured with the help of the Pair Spectrometer [15]. The calculated integrated luminosity (Fig. 2(a)) shows the coherent peaks and reflects the efficiencies of the tagger detectors. The yield of a highstatistics reaction $\gamma+p \rightarrow \rho^{0}+p$, normalized by the luminosity spectrum is shown in Fig. 2(b) and is smooth as expected.

(a)

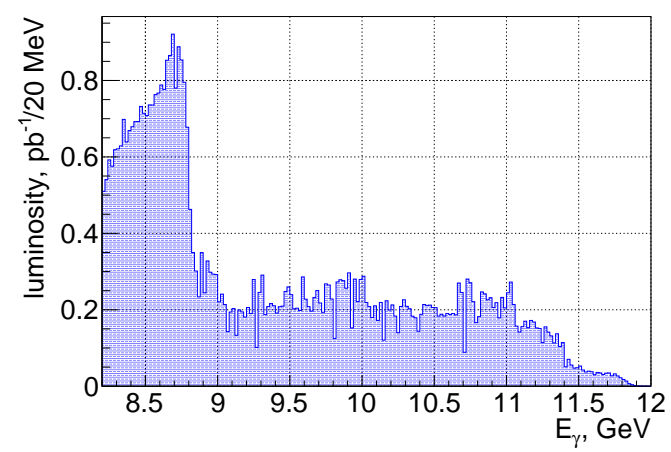

(b)

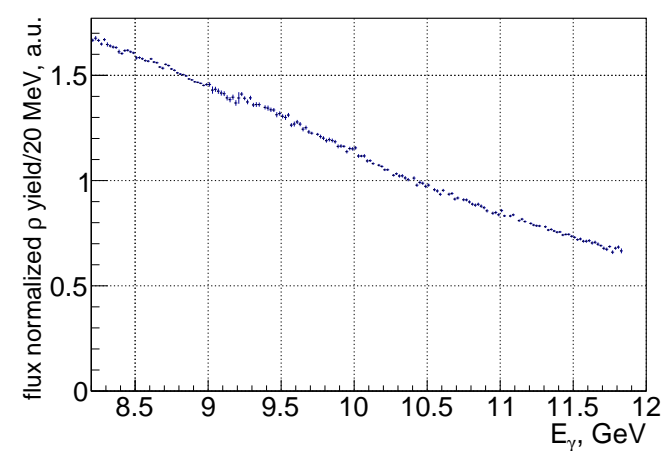

Figure 2: (a) - the energy spectrum of the integrated luminosity for the 2016 and 2017 data; (b) - the luminosity-normalized yield of the reaction $\gamma+p \rightarrow \rho^{0}+p$ (the 2016 data only) indicates that the beam flux normalization provides a smooth energy dependence for high-statistics reactions.

The photon beam interacts with a $30 \mathrm{~cm}$-long liquid hydrogen target located in the upstream part of the GlueX spectrometer in Hall D (Fig. 3). The spectrometer is based on a superconducting solenoid magnet with a bore $4 \mathrm{~m}$ long and $2 \mathrm{~m}$ in diameter. The magnet provides a $\sim 2 \mathrm{~T}$ field at the axis. The tracks of charged particles are detected with the Central Drift Chamber made of straw tubes and by the Forward Drift Chambers [16], all located in the bore of the solenoid. The photons are detected with the Barrel Calorimeter [17] located in the bore of the solenoid, and the Forward Calorimeter made of 2800 lead glass blocks located downstream of the solenoid. Scintillating hodoscopes around the target and in front of the Forward Calorimeter are used for timing measurements. The spectrometer has a uniform acceptance for charged particles and photons in a range of $0<\varphi<360^{\circ}, 1^{\circ}<\theta<120^{\circ}$. For the particle identification time-of-flight, $d E / d x$, and electromagnetic calorimeter techniques are used.

The typical photon flux was about $20 \mathrm{MHz}$ for $E_{\gamma}>8 \mathrm{GeV}$. The trigger logic selected events with the full energy in the electromagnetic calorimeters above a certain value, and had a high detection efficiency for the decay $J / \psi \rightarrow e^{+} e^{-}$. The DAQ rate was $20-50 \mathrm{kHz}$.

\subsection{Data Analysis}

The results presented are based on the data taken in 2016 and 2017, which accounts for $~ 20 \%$ of the full existing data set. The analysis included the reconstruction of charged tracks in the drift chambers and energy clusters in the calorimeters. We looked for the decay $J / \psi \rightarrow e^{+} e^{-}$. The candidates for the exclusive reaction $\gamma+p \rightarrow p+e^{+}+e^{-}$were selected using the kinematic fit of the 


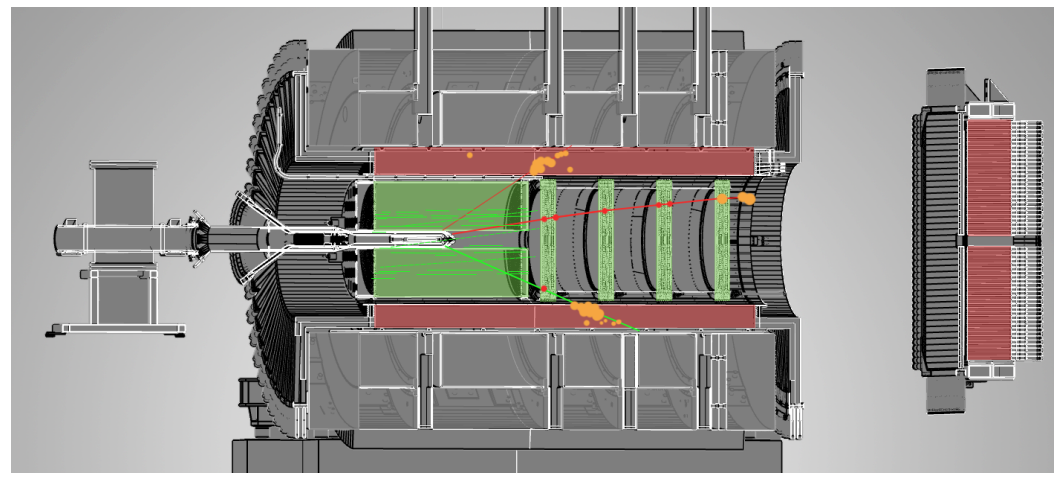

Figure 3: Hall D: the GlueX spectrometer.

final state particles with the measured energy of the beam photon. The typical timing resolutions of the spectrometer as well as the tagger detectors were much better than the time distance between the beam bunches $(4 \mathrm{~ns})$. The rate of accidental coincidences of the spectrometer events with the tagger signals was measured using the off-time tagger signals. The protons are well identifiable using the $d E / d x$ in the drift chambers and the TOF measurements. The electrons/positrons were identified against a large pion background by comparing their energy release $E$ in the electromagnetic calorimeters with the measured momentum $p$. The structure of the Barrel Calorimeter allowed also to employ the "pre-shower/shower" leverage. Selecting $e^{+} e^{-}$candidates with $p / E<1.15$ reduced the pion background by a factor of $\sim 5000$.

The mass spectrum of $e^{+} e^{-}$candidates, corrected for the accidental coincidences, is shown in Fig. 4. The $J / \psi$ signal and the $\phi$ signal are seen. The continuum consists of the Bethe-Heitler pairs as well as a $\pi^{+} \pi^{-}$background.

(a)

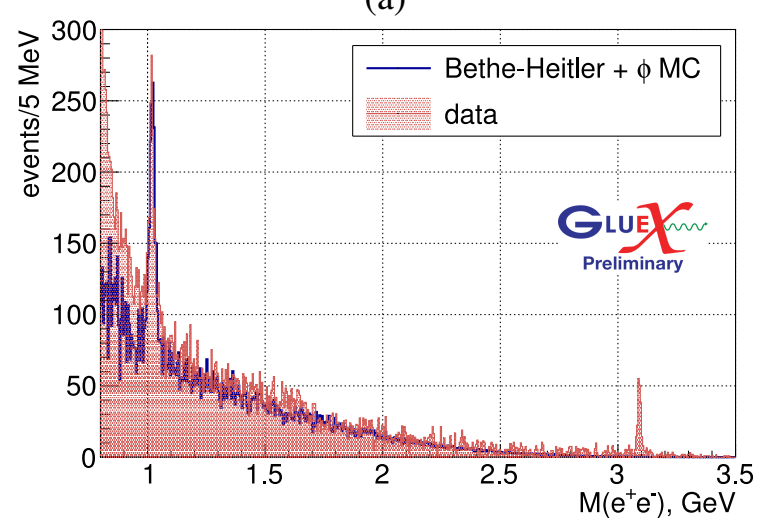

(b)

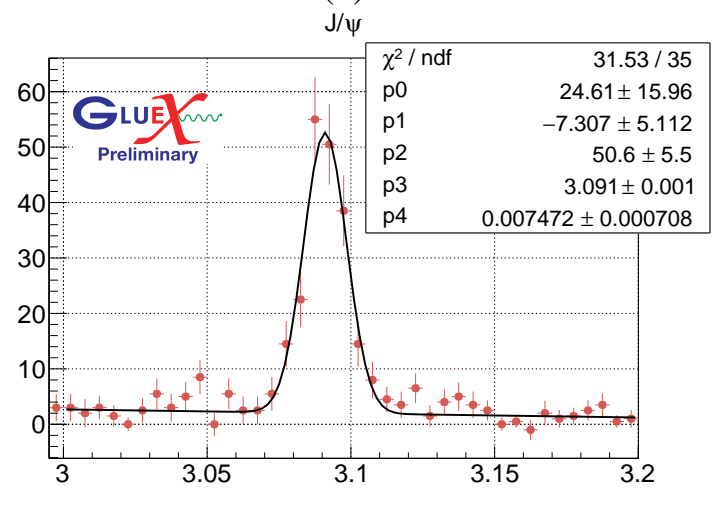

Figure 4: (a) - the mass spectrum of $e^{+} e^{-}$candidates. It shows the $\phi$ and $J / \psi$ peaks. The continuum consists of the Bethe-Heitler pairs as well as a $\pi^{+} \pi^{-}$background. (b) - the zoomed area of the $J / \psi$ signal. It contains $189 \pm 16$ candidates.

In order to evaluate the $J / \psi$ photoproduction cross section, we used for normalization the detected Bethe-Heitler process in the $1.5<M_{e^{+} e^{-}}<2.5 \mathrm{GeV} / \mathrm{c}^{2}$ interval (see Fig. 4(a)). The Bethe-Heitler Monte-Carlo simulation and the cross-section calculation were based on the work of Berger, Diehl, and Pire [18]. The beam energy range was split into 6 bins. In each bin the $J / \psi$ and 
the Bethe-Heitler signals $N_{J / \psi}, N_{B H}$, as well as the detection efficiencies $\varepsilon_{J / \psi}, \varepsilon_{B H}$ were estimated. The cross section was calculated as: $\sigma_{J / \psi}=\sigma_{B H} \cdot N_{J / \psi} / N_{B H} \cdot \varepsilon_{B H} / \varepsilon_{J / \psi}$. The relative level of the $\pi^{+} \pi^{-}$background in the Bethe-Heitler sample was about $40 \%$. It was measured in each energy bin, using half-identified pairs and the measured response of the electromagnetic calorimeters to pions. The results are shown in Fig. 5.

(a)

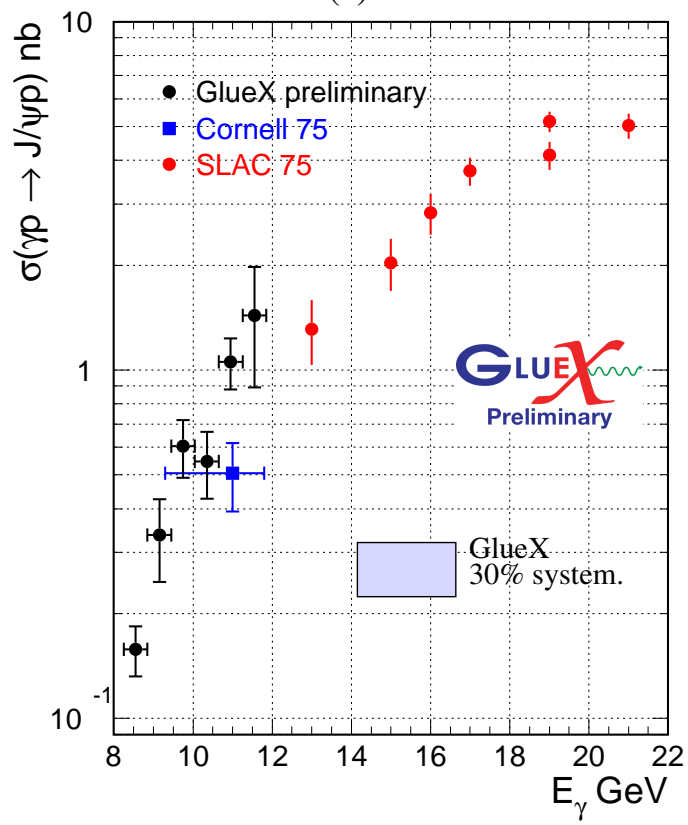

(b)

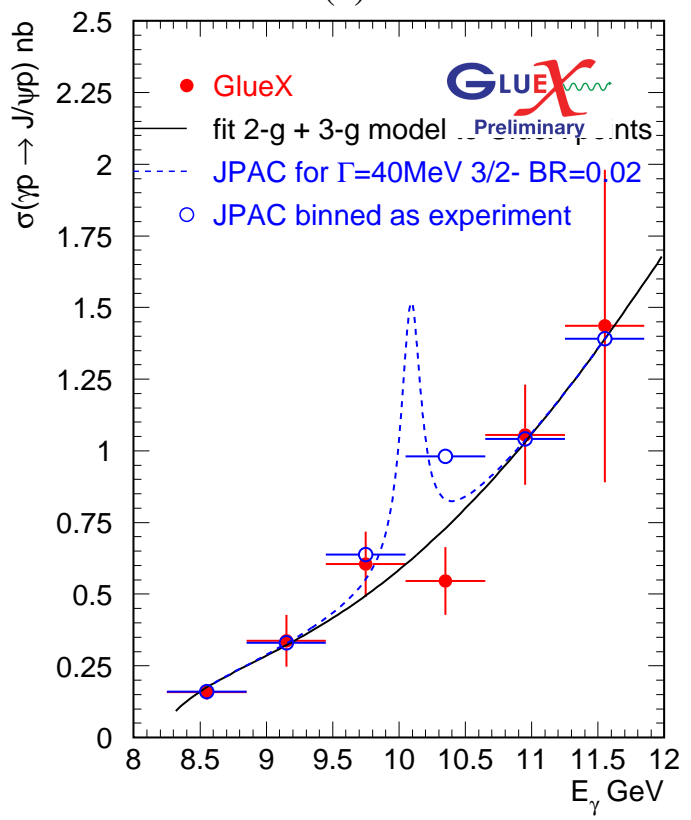

Figure 5: The energy dependence of the $J / \psi$ photoproduction cross section. (a) - the GlueX results. The error bars present the statistical errors. The systematic errors were evaluated at $30 \%$. A comparison with the 1975 data from SLAC [1] and Cornell [2] is shown. Those experiments measured the $\frac{d \sigma}{d t}=A \cdot e^{\beta t}$. In order to derive the full cross section we used $\beta=2.9(\mathrm{GeV} / \mathrm{c})^{-2}$ ([1]) and $1.25(\mathrm{GeV} / \mathrm{c})^{-2}$ ([2]). (b) - the GlueX results in a linear scale. The black solid line shows the result of a fit of a production model [4] to the GlueX data assuming no pentaquark contribution. The blue dash line shows a prediction [12] (obtained using the provided calculator [19]) for the pentaquark $P(4450)\left(\frac{3}{2}^{-}\right)$production, assuming $B R(P \rightarrow J / \psi p)=2 \%$ and the contribution from the $t$-channel equal to the result of the fit to the data (the black solid line). The blue open circles show the same prediction with the appropriate binning.

The GlueX results for the full cross section are higher than the old results from SLAC and Cornell (see Fig. 5(a)). In order to derive the full cross section from those old results we had to assume a certain $t$-dependence of the cross section. The GlueX data are well fit with a model [4] that has two free parameters for the contributions from the 2- and 3-gluon exchanges (see Fig. 5(b)). The second component is needed to describe the data below $10 \mathrm{GeV}$, where the first component falls too steeply to the threshold.

The experimental results are compared with a prediction [12] for the pentaquark $P(4450)\left(\frac{3}{2}^{-}\right)$, $\Gamma=39 \mathrm{MeV} / \mathrm{c}^{2}$ production. The prediction depends on the $t$-channel contribution (evaluated using this experimental result) and on the decay branching ratio $B R(P \rightarrow J / \psi p)$. For $B R=2 \%$ the experimental result in the two bins around the resonance $9.45<E_{\gamma}<10.65 \mathrm{GeV}$ is about $3 \sigma($ stat $)$ lower than the prediction. The systematic uncertainties of the prediction may come from several 
factors: the interference between the amplitudes of the $t$ - and $s$-channels; the VMD model; a potential influence of the wider LHCb pentaquark $P(4380), \Gamma=205 \mathrm{MeV} / \mathrm{c}^{2}$.

\section{Summary and Outlook}

The GlueX experiment has measured the cross section of the reaction $\gamma+p \rightarrow J / \psi+p$ in the energy interval $8.2<E_{\gamma}<11.8 \mathrm{GeV}$, which is close to the threshold of the reaction $(8.2 \mathrm{GeV})$. Preliminary results, based on $20 \%$ of the recorded data set, are presented.

The energy dependence of the cross section is compared with a theoretical model [4]. In the framework of this model the high energy $\left(E_{\gamma}>13 \mathrm{GeV}\right)$ measurements are well described by a 2-gluon exchange. The GlueX data at lower energy indicate a flatter slope and is better described by a combination of the 2- and 3-gluon exchanges.

The GlueX results show no statistically-significant evidence for a contribution from the narrow pentaquark $P(4450)\left(\frac{3}{2}^{-}\right), \Gamma=39 \mathrm{MeV} / \mathrm{c}^{2}$. The model-dependent [12] level of sensitivity is $B R(P \rightarrow J / \psi p) \sim 2 \%$. The systematic uncertainties of the model as well as of the experimental results remain to be addressed. The results can be improved in the future by considering the $t$ dependence of the cross section, expected to be different between the $t$-channel $J / \psi$ production and the $s$-channel pentaquark production and decay.

In a couple of months the GlueX collaboration is expected to analyze more data increasing the statistics by a factor of $\sim 2$. It may take another year to analyze the full data set.

\section{References}

[1] U. Camerini, J. G. Learned, R. Prepost, C. M. Spencer, D. E. Wiser, W. Ash et al., Photoproduction of the $\psi$ Particles, Phys. Rev. Lett. 35 (1975) 483.

[2] B. Gittelman, K. M. Hanson, D. Larson, E. Loh, A. Silverman and G. Theodosiou, Photoproduction of the $\psi(3100)$ Meson at 11-GeV, Phys. Rev. Lett. 35 (1975) 1616.

[3] D. Kharzeev, H. Satz, A. Syamtomov and G. Zinovjev, J/psi photoproduction and the gluon structure of the nucleon, Eur. Phys. J. C9 (1999) 459 [hep-ph/9901375].

[4] S. J. Brodsky, E. Chudakov, P. Hoyer and J. M. Laget, Photoproduction of charm near threshold, Phys. Lett. B498 (2001) 23 [hep-ph/ 0010343$].$

[5] L. Frankfurt and M. Strikman, Two-gluon form factor of the nucleon and j/psi photoproduction, Phys. Rev. D66 (2002) 031502 [hep-ph/ 0205223$].$

[6] A. Sibirtsev, S. Krewald and A. W. Thomas, Systematic analysis of charmonium photoproduction, J. Phys. G30 (2004) 1427.

[7] LHCB collaboration, R. Aaij et al., Observation of $J / \psi p$ Resonances Consistent with Pentaquark States in $\Lambda_{b}^{0} \rightarrow J / \psi K^{-} p$ Decays, Phys. Rev. Lett. 115 (2015) 072001 [1507.03414].

[8] Q. Wang, X.-H. Liu and Q. Zhao, Photoproduction of hidden charm pentaquark states $P_{c}^{+}(4380)$ and $P_{c}^{+}$(4450), Phys. Rev. D92 (2015) 034022 [1508.00339].

[9] V. Kubarovsky and M. B. Voloshin, Formation of hidden-charm pentaquarks in photon-nucleon collisions, Phys. Rev. D92 (2015) 031502 [1508.00888]. 
[10] M. Karliner and J. L. Rosner, Photoproduction of Exotic Baryon Resonances, Phys. Lett. B752 (2016) 329 [1508.01496].

[11] M. I. Eides, V. Yu. Petrov and M. V. Polyakov, Narrow Nucleon- $\psi(2 S)$ Bound State and LHCb Pentaquarks, Phys. Rev. D93 (2016) 054039 [1512. 00426 ].

[12] A. N. Hiller Blin, C. Fernández-Ramírez, A. Jackura, V. Mathieu, V. I. Mokeev, A. Pilloni et al., Studying the $P_{c}(4450)$ resonance in J/ $\psi$ photoproduction off protons, Phys. Rev. D94 (2016) 034002 [1606.08912].

[13] M. I. Eides, V. Yu. Petrov and M. V. Polyakov, Pentaquarks with hidden charm as hadroquarkonia, Eur. Phys. J. C78 (2018) 36 [1709.09523].

[14] J. Dudek, R. Ent, R. Essig, K. S. Kumar, C. Meyer, R. D. McKeown et al., Physics opportunities with the 12 gev upgrade at jefferson lab, The European Physical Journal A 48 (2012) 187.

[15] F. Barbosa, C. Hutton, A. Sitnikov, A. Somov, S. Somov and I. Tolstukhin, Pair spectrometer hodoscope for Hall D at Jefferson Lab, Nucl. Instrum. Meth. A795 (2015) 376.

[16] L. Pentchev, F. Barbosa, V. Berdnikov, D. Butler, S. Furletov, L. Robison et al., Studies with cathode drift chambers for the GlueX experiment at Jefferson Lab, Nucl. Instrum. Meth. A845 (2017) 281.

[17] T. D. Beattie et al., Construction and Performance of the Barrel Electromagnetic Calorimeter for the GlueX Experiment, Nucl. Instrum. Meth. A896 (2018) 24 [1801.03088].

[18] E. R. Berger, M. Diehl and B. Pire, Time - like Compton scattering: Exclusive photoproduction of lepton pairs, Eur. Phys. J. C23 (2002) 675 [hep-ph/ 0110062 ].

[19] A. N. Hiller Blin, C. Fernández-Ramírez, A. Jackura, V. Mathieu, V. I. Mokeev, A. Pilloni et al., “Calculator for $\gamma p \rightarrow J / \psi p$ reaction.” http://cgl.soic.indiana.edu/jpac/PentaQ_JPsi.php, 2016. 